\title{
Proactive Population Health Strategy to Offer Tobacco Dependence Treatment to Smokers in a Primary Care Practice Network
}

\author{
Sara Kalkhoran, MD, MAS ${ }^{1,2,3}$, Elizabeth $M$. Inman, BA ${ }^{1,4}$, Jennifer H. K. Kelley, RN, MA $A^{1,4}$, \\ Jeffrey M. Ashburner, $P h D, M P H^{2,3}$, and Nancy A. Rigotti, MD ${ }^{1,2,3,4}$
}

\begin{abstract}
'Tobacco Research and Treatment Center, Massachusetts General Hospital, Boston, MA, USA; ${ }^{2}$ Division of General Internal Medicine, Department of Medicine, Massachusetts General Hospital, Boston, MA, USA; ${ }^{3}$ Harvard Medical School, Boston, MA, USA; ${ }^{4}$ Mongan Institute Health Policy Center, Massachusetts General Hospital, Boston, MA, USA.
\end{abstract}

BACKGROUND: Population-based strategies can expand the reach of tobacco cessation treatment beyond clinical encounters.

OBJECTIVE: To determine the effect of two populationbased tobacco cessation strategies, compared with usual care, on providing tobacco treatment outside of clinical encounters.

DESIGN: 3-arm pragmatic randomized controlled trial.

PARTICIPANTS: Current smokers $\geq 18$ years old with a primary care provider at one of five community health centers in Massachusetts were identified via the electronic health record $(n=5225)$ and recruited using automated phone calls.

INTERVENTIONS: One intervention group involved engagement with a health system-based tobacco coach (internal care coordination), and the other connected patients to a national quitline (external community referral). MEASUREMENTS: Proportion of smokers with documentation of any evidence-based cessation treatment in the 6 months after enrollment.

KEY RESULTS: Of 639 individuals who responded to the proactive treatment offer, 233 consented and were randomized 1:1:1 to study arm. At 6-month follow-up, the pooled intervention group, compared with usual care, had higher documentation of any smoking cessation treatment (63\% vs. 34\%, $p<0.001$ ), cessation medication prescription (52\% vs. $30 \%, p=0.002)$, and counseling $(47 \%$ vs. $9 \%, p<0.001)$. Internal care coordination was more effective than external community referral at connecting smokers to any cessation treatment $(76 \%$ vs. $50 \%, p=$ 0.001 ) and at providing cessation medication (66\% vs. $39 \%, p<0.001)$, but comparable at linking smokers to cessation counseling resources.

CONCLUSIONS: Smokers responding to a populationbased, proactive outreach strategy had better provision of tobacco cessation treatment when referred to either a

$\overline{\text { This study was presented at the } 2018 \text { Society for General Internal }}$ Medicine Annual Meeting in Denver, $\mathrm{CO}$.

Electronic supplementary material The online version of this article (https://doi.org/10.1007/s11606-019-05079-3) contains supplementary material, which is available to authorized users.

Received August 15, 2018

Revised February 7, 2019

Accepted April 23, 2019

Published online June 13, 2019 health system-based or community-based program compared with usual care. The health system-based strategy outperformed the quitline-based one in several measures. Future work should aim to improve population reach and test the effect on smoking cessation rates.

TRIAL REGISTRATION: ClinicalTrials.gov NCT03612895

KEY WORDS: smoking cessation; primary care; population health; clinical trials.

J Gen Intern Med 34(8):1571-7

DOI: $10.1007 / \mathrm{s} 11606-019-05079-3$

(c) Society of General Internal Medicine 2019

\section{INTRODUCTION}

Smoking remains the leading preventable cause of death in the USA. ${ }^{1}$ An estimated 36.5 million US adults, or $15 \%$, smoke cigarettes. $^{2}$ While effective tobacco cessation treatments exist, ${ }^{3}$ in 2015 , only $31 \%$ of smokers who tried to quit in the past year reported having used any assistance. ${ }^{4}$ Healthcare systems are a major channel for delivering tobacco cessation treatment resources to smokers, but current systems often fail to offer treatment reliably. ${ }^{5}$ One reason is that health systems primarily use reactive strategies that rely on busy clinicians with multiple competing demands to provide treatment at office visits.

Offering treatment independent of clinical encounters is a population-based strategy that could expand the reach of tobacco cessation treatment in health systems by complementing clinicians' office-based efforts. ${ }^{6,7}$ Electronic health records (EHR) facilitate creation of population-based registries of current smokers to whom tobacco treatment can be proactively offered independent of clinical visits. ${ }^{6}$ The effectiveness of this approach is supported by five randomized controlled trials in which smokers who were offered proactive evidence-based treatment had higher smoking cessation rates at follow-up than smokers receiving usual care. ${ }^{8-12}$ The trials all leveraged the EHR to identify a population of current smokers and reached out to smokers using mailed letters or automated or live telephone calls. To provide treatment to smokers who engaged, all trials offered telephone-based cessation counseling and facilitated smokers' access to cessation medications. In 
each trial, however, telephone counseling was delivered by research staff, and in three trials, research funds provided free nicotine replacement therapy. For a proactive tobacco treatment strategy to be broadly implemented, it must be sustainable in real-world healthcare settings where resources are often limited.

A key challenge for health systems is to define what services to provide to smokers responding to proactive treatment offers. The landscape of tobacco treatment services suggests two alternatives: internal health system-based resources or external community-based resources, such as the national network of telephone quitlines. ${ }^{13}$ An internal program provides closer coordination with the smokers' healthcare team and might therefore be more effective than a quitline referral. However, a quitline referral incurs no cost to the health system because quitline services are primarily funded by state health departments. The effectiveness of these two approaches for delivering treatment in a proactive care model has not been directly compared.

We conducted a pragmatic randomized controlled trial on the effect of two proactive, population-based outreach strategies, compared with usual care, for providing tobacco cessation treatment to smokers outside of clinical encounters. The trial design permitted us to compare an internal tobacco care coordination strategy to an external community-based referral strategy and compare both to usual care. We hypothesized that the interventions would have higher rates of connecting current smokers to tobacco treatment, and that the internal care coordination strategy would connect more smokers to treatment than quitline referral.

\section{METHODS}

\section{Design}

Be Tobacco-Free (BTF) was a three-arm pragmatic randomized controlled trial comparing the efficacy of two populationbased, proactively offered interventions for delivering tobacco cessation treatment vs. usual care. The Partners Institutional Review Board approved the study. Enrollment began in April 2016 and outcome ascertainment ended in August 2017.

\section{Setting and Participants}

We recruited participants from five Massachusetts General Hospital (MGH)-affiliated primary care practices located at community health centers. Using the EHR, we identified adults ( $\geq 18$ years old) who were seen at a participating practice in the past year, had a current smoking status, and had a telephone number. We excluded patients who did not speak English and those who had a problem list diagnosis of dementia, psychosis, schizophrenia, schizoaffective disorder, or suicidal tendencies. Primary care physicians (PCPs) were provided brief information about the study along with a request to permit study staff to contact their potentially eligible patients for participation. Anyone they deemed inappropriate to contact was excluded.

Eligible smokers received a letter, signed by their PCP and the study's principal investigator, describing the study and providing contact information to opt out of future contact. Smokers who did not opt out received an automated telephone call using interactive voice response (IVR) technology (TelASK Technologies, Ottawa, Canada). The call described the study, confirmed eligibility, obtained oral informed consent, administered a brief survey about smoking history, and randomized participants. Up to 12 call attempts were made over 2 weeks. IVR technology was chosen because of greater efficiency compared with live calls and its acceptability for participant recruitment. ${ }^{10,14}$

\section{Randomization and Intervention}

We randomized participants $1: 1: 1$, stratified by practice, to one of two intervention groups (internal care coordination or external community referral) or usual care. A statistician using a random number generator with blocks created the random allocation sequence. The IVR system implemented the randomization table for patients who consented and completed baseline data collection. Participants were unblinded to study arm.

The core component of internal care coordination was a MGH-based Tobacco Care Coordinator-introduced to participants as the MGH tobacco coach. The coach called each new enrollee to assess tobacco use and readiness to quit, offer brief counseling and motivational intervention, describe tobacco treatment options, and help them make a quit plan. The coach linked participants to their choice of internal (healthcare system-based) and external (community-based) tobacco cessation resources. Internal resources included referral to practice-based in-person tobacco counseling and communication with the PCP to facilitate tobacco cessation medication prescriptions. External resources included fax-referral to the Massachusetts Smoker's Quitline $^{13}$ and assistance signing up for the SmokeFreeTXT text messaging program. ${ }^{15}$ The coach documented actions taken as an EHR note. IVR telephone calls were made 4, 8, and 12 weeks after enrollment, each offering a call back from the coach for counseling or medication assistance. If the IVR system did not reach a participant for a scheduled call, the coach made up to three attempts to call the participant.

In the external community referral group, participants were connected at the end of the IVR recruitment call directly to the Massachusetts Smoker's Quitline via a warm transfer. The quitline offered up to five proactive telephone counseling calls over 12 weeks with options for text messaging, web-based resources, mailed educational materials, and information about insurance coverage of smoking cessation medications. It also mailed a 4-week supply of free nicotine patches, gum, or lozenges to participants setting a quit date within 30 days.

Participants in the usual care group were given the state quitline number at randomization and advised to contact their PCP for assistance to quit smoking. Each practice had a 
certified tobacco treatment specialist available one day per week to provide free in-person individual cessation support. PCPs could also fax a referral to the quitline.

\section{Measures}

Baseline measures collected from the EHR included demographics (age, sex, race, education level), health insurance type, medical history (coronary heart disease, chronic obstructive pulmonary disease, diabetes, hypertension, asthma, depression), past-year prescription of tobacco cessation pharmacotherapy, and number of visits to the practice in the past three years. A baseline survey during the initial IVR call collected additional information about recent use of cigarettes, electronic cigarettes, and other tobacco products; cigarettes smoked per day; nicotine dependence (time to first morning cigarette ${ }^{16}$ ); past-year quit attempts; and intentions to quit in the next 30 days.

The primary outcome measure was the proportion of smokers with documentation of any evidence-based tobacco cessation treatment (counseling or pharmacotherapy) in the 6 months after enrollment. Any tobacco cessation treatment was defined as any of the following: (1) in-person visit with the practice-based tobacco counselor; (2) completion of $\geq 1$ quitline counseling call (excluding the initial registration call to which the participant was transferred from the IVR system); (3) telephone contact with the study's tobacco coach (excluding the initial coordination call at enrollment); (4) outpatient prescription for nicotine replacement, bupropion, or varenicline in the EHR; or (5) provision of nicotine replacement by the quitline. Information was obtained from the EHR, research program records, and practice-based tobacco counselor records by unblinded study staff. The quitline provider, National Jewish Health, provided patient-level data on all quitline referrals sent from all MGH practices during the study.

In exploratory analyses, we measured self-reported past 30day tobacco abstinence at 6 months after enrollment, using an IVR-administered survey. Up to 12 IVR calls were made at different times, and participants who did not complete IVR calls were called three times by study staff and sent an email prompt to complete the survey.

\section{Statistical Analysis}

We compared participants' baseline characteristics by study group using chi-square tests, $t$ tests, and Fisher's exact tests. The primary outcome analysis, conducted as intention-to-treat, compared the pooled intervention groups (internal care coordination and external community referral) to usual care, using chi-square tests. In secondary analyses, we compared the two intervention groups to each other and each to usual care. In exploratory analyses, we compared groups on self-reported past 30-day tobacco abstinence at 6 months, using both a complete case analysis that included only participants who completed the 6-month survey and an analysis that counted survey non-respondents as smokers. We used SAS version 9.4 for all analyses.

\section{RESULTS}

Using EHR data, we identified 5890 smokers who were potentially eligible for the study (Fig. 1) and mailed recruitment letters to the 5654 smokers (96\%) who were not excluded by their PCPs. After excluding smokers who opted out of further study communication, were ineligible for the study, and were unreachable by mail, 5199 smokers $(88 \%)$ were contacted by IVR for enrollment. Of the 639 individuals (12\%) who answered the IVR call, 198 (31\%) denied being current smokers. Among the 441 eligible smokers who answered the IVR call, 155 refused study participation, 4 calls were disconnected, and $282(64 \%)$ consented to join the study. Due to technical problems, 49 failed authentication before randomization and 233 participants were randomly assigned to the internal care coordination group $(n=79)$, the external community referral group $(N=78)$, or to the usual care group $(n=76)$. Overall, $5.4 \%(n=282)$ of the 5199 individuals who received IVR calls consented to join the study and $4.5 \%(n=233)$ were successfully randomized. Characteristics of individuals who did and did not respond to the IVR call are shown in the online appendix (Supplemental Table 1).

Compared with eligible individuals who did not enroll in the study, participants who enrolled were older (mean age 53.4 vs. 49.3$)$; more likely to be female ( $63.5 \%$ vs. $52.4 \%)$, black ( $9.4 \%$ vs. $4.4 \%$ ), have Medicare insurance ( $30.3 \%$ vs. $21.3 \%$ ), have COPD (44.6\% vs. $34.9 \%)$ and depression (10.3\% vs. $6.6 \%$ ), and have used smoking cessation medication in the past year (43.4\% vs. $24.1 \%)$; and less likely to be Hispanic $(4.7 \%$ vs. $9.1 \%)$ (all $p$ values $<0.05)$.

Participants in the three study groups had similar baseline characteristics (Table 1). The internal care coordination group had a higher prevalence of coronary artery disease compared with the external community referral $(p=0.05)$ and usual care groups $(p=0.05)$. No other factors differed significantly across groups.

Table 2 shows results of the primary analyses assessing participants' documented receipt of evidence-based tobacco cessation treatment over 6-month follow-up. The pooled intervention group was nearly twice as likely as the usual care group to have documentation of any evidence-based cessation treatment $(63.1 \%$ vs. $34.2 \%, p<0.001)$. The pooled intervention group also had more documentation of counseling ( $46.5 \%$ vs. $9.2 \%, p<0.001)$, smoking cessation medication $(52.2 \%$ vs. $30.3 \%, p=0.002$ ), and both counseling and medication $(35.7 \%$ vs. $5.3 \%, p<0.001)$ than usual care.

Secondary analyses compared the two intervention groups with each other and with usual care (Table 3 ). The internal care coordination group was more likely than the external community referral group to have documented receipt of any tobacco cessation treatment and cessation medication provision ( $76.0 \%$ vs. $50.0 \%, p=0.001$ ). The groups did not differ in receipt of counseling or of both counseling and medication. However, use of specific counseling resources varied by group. The internal care coordination group had more in- 


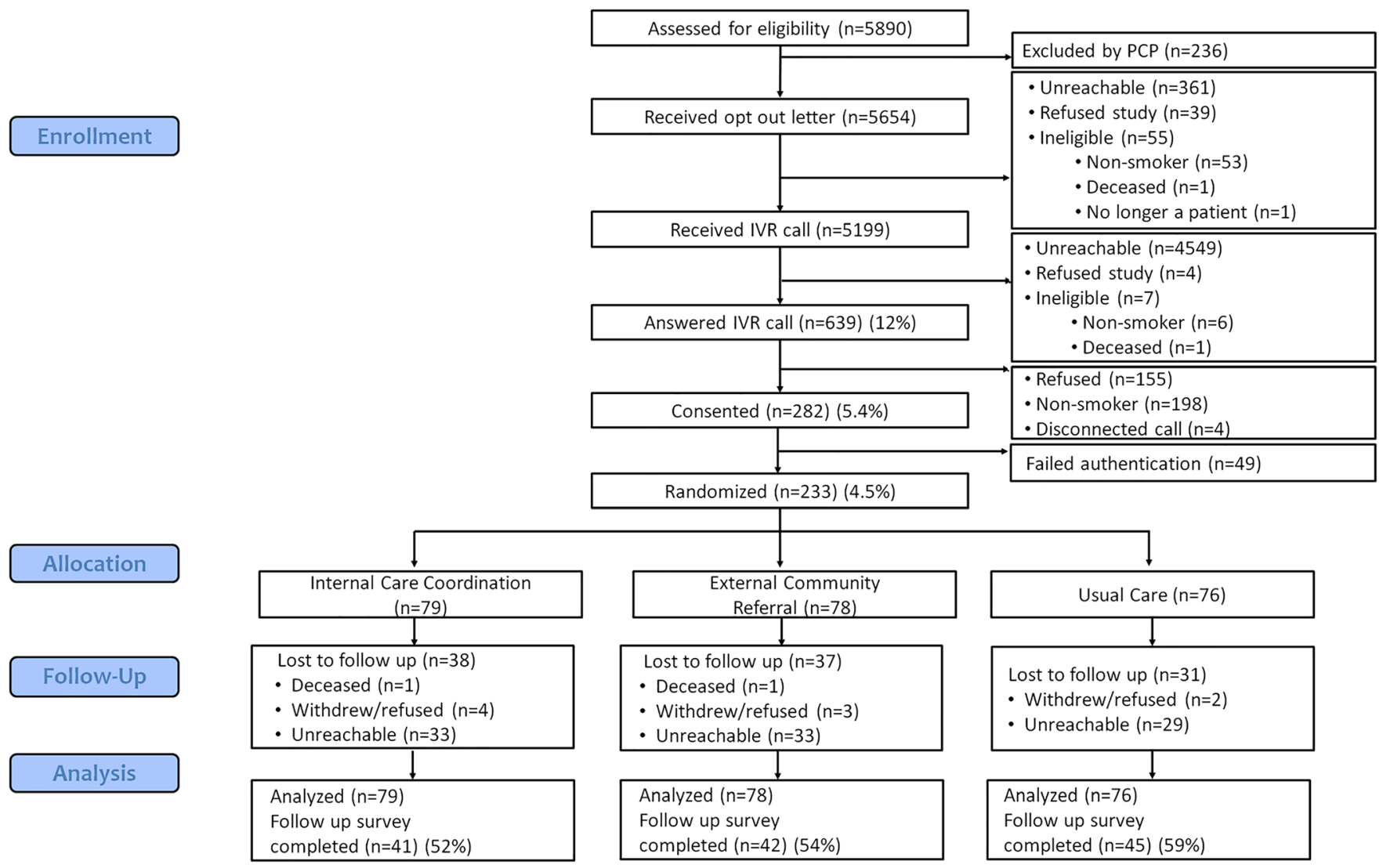

Figure 1 Consort diagram.

person counseling and less quitline counseling than the external community referral group $(22.8 \%$ vs. $3.8 \%, p<0.001$ for in-person counseling; $15.2 \%$ vs. $41.8 \%, p<0.001$ for quitline counseling). Each intervention group was more likely than the usual care group to have documented receipt of counseling, but only the internal care coordination group outperformed the usual care group in providing cessation medication (Table 3 ).

We conducted exploratory analyses of self-reported smoking status at 6-month follow-up. The survey response rate was $54.7 \%$ overall and did not differ significantly by study $\operatorname{arm}(p=0.64$, Fig. 1). In the complete case analysis $(N=128)$, self-reported past 30-day cigarette abstinence was $14.6 \%$ in the internal care coordination group, $14.3 \%$ in the external community referral group, and $8.9 \%$ in the usual care group. Counting non-respondents as smokers, self-reported past 30day cigarette abstinence rates were $7.6 \%$ in the internal care coordination group, $7.7 \%$ in the external community referral group, and $5.3 \%$ in the usual care group. While rates were higher in the two intervention groups, these differences were not statistically significant.

\section{DISCUSSION}

In this pragmatic randomized controlled trial conducted at five community health centers, the proactive offer of tobacco cessation treatment to a population of current smokers identified via the EHR and contacted independent of office visits was more effective than usual care in connecting smokers to evidence-based treatment. The trial further compared the effectiveness of two models of tobacco cessation treatment provision in this setting. A care coordination model based in the healthcare system was more effective than an external referral to community-based telephone quitline for connecting smokers to any documented tobacco cessation treatment and at increasing the provision of cessation medication. The two models were comparable at linking smokers to counseling resources, though the specific resources varied in a predictable way. Participants referred to the quitline primarily received counseling from the quitline, while those referred to the tobacco care coordinator had more contacts with internal counseling resources. Nevertheless, each intervention was more effective than usual care at linking smokers to combined counseling and medication, the most effective strategy for long-term cessation. ${ }^{3}$

The study findings are consistent with prior studies that also used a proactive outreach model ${ }^{8-11,17}$ to offer tobacco cessation treatment to a population. Our study extends previous work by primarily using existing treatment resources to deliver cessation counseling and medication. ${ }^{8,10-12}$ For example, unlike prior studies, we did not use research funds to provide tobacco cessation medication. By delivering cessation treatment with minimal (as in the internal care coordination group) or no (as in the external community referral group) reliance on research staff, our treatment models may therefore be more 
Table 1 Baseline Sociodemographic and Tobacco Use Characteristics of the Study Population

\begin{tabular}{|c|c|c|c|c|}
\hline & $\begin{array}{l}\text { Internal care } \\
\text { coordination }(n=79)\end{array}$ & $\begin{array}{l}\text { External community } \\
\text { referral }(n=78)\end{array}$ & $\begin{array}{l}\text { Pooled intervention } \\
\text { groups }(n=157)\end{array}$ & Usual care $(n=76)$ \\
\hline Age, mean (SD) & $53.7(11.9)$ & $52.9(12.2)$ & $53.3(12.0)$ & $53.5(11.3)$ \\
\hline Clinic visits over 3 years, mean (SD) & $12.6(9.1)$ & $12.3(11.0)$ & $12.5(10.1)$ & $10.4(7.4)$ \\
\hline Female sex & $54(68.4 \%)$ & $51(65.4 \%)$ & $105(66.9 \%)$ & $43(56.6 \%)$ \\
\hline \multicolumn{5}{|l|}{ Race } \\
\hline Non-Hispanic White & $65(82.3 \%)$ & $57(73.1 \%)$ & $122(77.7 \%)$ & $61(80.3 \%)$ \\
\hline Hispanic & $3(3.8 \%)$ & $5(6.4 \%)$ & $8(5.1 \%)$ & $3(4.0 \%)$ \\
\hline Non-Hispanic Black & $5(6.3 \%)$ & $10(12.8 \%)$ & $15(9.6 \%)$ & $7(9.2 \%)$ \\
\hline Non-Hispanic Asian & $1(1.3 \%)$ & $1(1.3 \%)$ & $2(1.3 \%)$ & $0(0.0 \%)$ \\
\hline Other/unknown & $5(6.3 \%)$ & $5(6.4 \%)$ & $10(6.4 \%)$ & $5(6.6 \%)$ \\
\hline \multicolumn{5}{|l|}{ Insurance } \\
\hline Commercial & $39(49.4 \%)$ & $41(52.6 \%)$ & $80(51.0 \%)$ & $41(54.0 \%)$ \\
\hline Medicaid & $15(19.0 \%)$ & $9(11.5 \%)$ & $24(15.3 \%)$ & $8(10.5 \%)$ \\
\hline Medicare & $22(27.9 \%)$ & $24(30.8 \%)$ & $46(29.3 \%)$ & $24(31.6 \%)$ \\
\hline Uninsured & $3(3.8 \%)$ & $4(5.1 \%)$ & $7(4.5 \%)$ & $3(4.0 \%)$ \\
\hline \multicolumn{5}{|l|}{ Education } \\
\hline Less than high school & $4(5.1 \%)$ & $2(2.6 \%)$ & $6(3.8 \%)$ & $7(9.2 \%)$ \\
\hline High school & $33(41.8 \%)$ & $21(26.9 \%)$ & $54(34.4 \%)$ & $23(30.3 \%)$ \\
\hline College graduate & $13(16.5 \%)$ & $10(12.8 \%)$ & $23(14.7 \%)$ & $15(19.7 \%)$ \\
\hline Unknown & $29(36.7 \%)$ & $45(57.7 \%)$ & $74(47.1 \%)$ & $31(40.8 \%)$ \\
\hline \multicolumn{5}{|l|}{ Medical diagnoses } \\
\hline COPD & $37(46.8 \%)$ & $35(44.9 \%)$ & $72(45.9 \%)$ & $32(42.1 \%)$ \\
\hline Asthma & $23(29.1 \%)$ & $19(24.4 \%)$ & $42(26.8 \%)$ & $17(22.4 \%)$ \\
\hline Coronary artery disease & $10(12.7 \%) *$ & $3(3.9 \%)$ & $13(8.3 \%)$ & $3(4.0 \%)$ \\
\hline Diabetes & $12(15.2 \%)$ & $12(15.4 \%)$ & $24(15.3 \%)$ & $10(13.2 \%)$ \\
\hline Hypertension & $35(44.3 \%)$ & $25(32.1 \%)$ & $60(38.2 \%)$ & $27(35.5 \%)$ \\
\hline Depression & $10(12.7 \%)$ & $9(11.5 \%)$ & $19(12.1 \%)$ & $5(6.6 \%)$ \\
\hline Past 7-day cigarette use & $79(100 \%)$ & $76(97.4 \%)$ & $155(98.7 \%)$ & $74(97.4 \%)$ \\
\hline Cigarettes/day, mean (SD) & $15.0(8.1)$ & $14.7(10.6)$ & $14.9(9.4)$ & $15.9(8.3)$ \\
\hline First cigarette within 30 min of waking & $63(79.8 \%)$ & $59(75.6 \%)$ & $122(77.7 \%)$ & $61(80.3 \%)$ \\
\hline Past 30-day e-cigarette use & $7(8.9 \%)$ & $6(7.7 \%)$ & $13(8.3 \%)$ & $8(10.5 \%)$ \\
\hline Past 30-day other tobacco use & $6(7.6 \%)$ & $5(6.4 \%)$ & $11(7.0 \%)$ & $4(5.3 \%)$ \\
\hline Past year quit attempt & $49(62.0 \%)$ & $48(61.5 \%)$ & $97(61.8 \%)$ & $54(71.1 \%)$ \\
\hline Plan to quit in next 30 days & $62(78.5 \%)$ & $57(75.0 \%)$ & $119(76.8 \%)$ & $57(75.0 \%)$ \\
\hline \multicolumn{5}{|c|}{ Smoking cessation medication prescription in past year } \\
\hline Nicotine patch & $12(15.2 \%)$ & $21(26.9 \%)$ & $33(21.0 \%)$ & $19(25.0 \%)$ \\
\hline Nicotine lozenge & $5(6.3 \%)$ & $7(9.0 \%)$ & $12(7.6 \%)$ & $8(10.5 \%)$ \\
\hline Nicotine gum & $3(3.8 \%)$ & $3(3.9 \%)$ & $6(3.8 \%)$ & $3(4.0 \%)$ \\
\hline Nicotine inhaler & $6(7.6 \%)$ & $3(3.9 \%)$ & $9(5.7 \%)$ & $4(5.3 \%)$ \\
\hline Bupropion & $13(16.5 \%)$ & $12(15.4 \%)$ & $25(15.9 \%)$ & $9(11.8 \%)$ \\
\hline Varenicline & $8(10.1 \%)$ & $6(7.7 \%)$ & $14(8.9 \%)$ & $8(10.5 \%)$ \\
\hline
\end{tabular}

Data on sociodemographics, comorbidities, and smoking cessation prescriptions were obtained from the electronic health record. Tobacco use variables were obtained by participant self-report

*Incidence of CAD was significantly higher in the internal care coordination group vs. the external community referral group ( $p=0.04)$ and vs. the usual care group $(p=0.05)$

easily implemented by health systems. Additionally, our study provides health systems with the first direct comparison between two models of treatment delivery in the context of population-based proactive outreach. This information can guide policymakers and health system administrators who are considering how best to implement tobacco cessation treatment. The internal care coordination model was superior to external community referral in linking smokers to any treatment, any medication, and a broader range of medications beyond NRT (Table 3). However, the models did not differ significantly in providing smokers with both counseling and medication, and each model surpassed usual care in connecting smokers to treatment. We did not measure treatment costs directly, but the quitline referral option should be less costly to healthcare systems because most do not currently pay for quitlines, whereas a health system would need to fund a care coordinator to link smokers to existing internal and external tobacco cessation resources. Care coordinators for tobacco use can serve an important role in the chronic care model of care, components of which include support for disease self-management, decision support systems, clinical information systems, and community-based resources. ${ }^{18}$ Studies in other chronic diseases, such as diabetes, have found that chronic care model interventions can improve outcomes. ${ }^{19,20}$ Elements of such interventions can be adopted when designing tobacco-based interventions.

In exploratory analysis, each intervention group generated a higher self-reported smoking cessation rate at 6 months than usual care $(7.6 \%$ and $7.7 \%$ vs. $5.1 \%)$, but the difference was not statistically significant. Small sample sizes limited statistical power for this analysis. However, odds of past 30-day abstinence at 6 months in this study $(\mathrm{OR}=1.8)$ are comparable with point-prevalence abstinence measures reported by prior studies that detected an effect of proactive intervention vs. usual care on smoking cessation outcomes. ${ }^{8-10,12}$ Our study's absolute quit rates were lower than those found in these prior studies, partly attributable to our lower survey response 
Table 2 Documented Use of Evidence-Based Cessation Treatment in Pooled Intervention and Usual Care Groups

\begin{tabular}{|c|c|c|c|}
\hline & $\begin{array}{l}\text { Pooled intervention } \\
\text { groups* }(n=157)\end{array}$ & Usual care group $(n=76)$ & $P$ value \\
\hline Any evidence-based tobacco cessation treatment & $99(63.1 \%)$ & $26(34.2 \%)$ & $<0.001$ \\
\hline Use of both evidence-based tobacco cessation counseling and medication & $56(35.7 \%)$ & $4(5.3 \%)$ & $<0.001$ \\
\hline Any counseling & $73(46.5 \%)$ & $7(9.2 \%)$ & $<0.001$ \\
\hline In-person counseling at MGH Health Center & $21(13.4 \%)$ & $7(9.2 \%)$ & 0.36 \\
\hline Telephone counseling by Quitline & $44(28.0 \%)$ & $0(0 \%)$ & $<0.001$ \\
\hline Telephone counseling by MGH Tobacco Care Coordinator & $29(18.5 \%)^{b}$ & $-\varphi^{\dagger}$ & - \\
\hline Any medication & $82(52.2 \%)$ & $23(30.3 \%)$ & 0.002 \\
\hline Nicotine replacement therapy (any) & $72(45.9 \%)$ & $18(23.7 \%)$ & 0.001 \\
\hline Nicotine patch & $40(25.5 \%)$ & $15(19.7 \%)$ & 0.33 \\
\hline Nicotine lozenge & $12(7.6 \%)$ & $5(6.6 \%)$ & 0.77 \\
\hline Nicotine gum & $14(8.9 \%)$ & $2(2.6 \%)$ & 0.08 \\
\hline Nicotine inhaler & $12(7.6 \%)$ & $1(1.3 \%)$ & 0.07 \\
\hline Bupropion & $16(10.2 \%)$ & $8(10.5 \%)$ & 0.94 \\
\hline Varenicline & $16(10.2 \%)$ & $4(5.3 \%)$ & 0.21 \\
\hline
\end{tabular}

*Includes 79 participants in the internal care coordination group and 78 participants in the external community referral group

${ }^{\dagger}$ Only participants in the internal care coordination group had access to the MGH Tobacco Coordinator

rate. The complete case analysis generated comparable cessation rates to prior studies.

Our population-level strategy offered cessation resources to all patients, regardless of interest in quitting or recent engagement in care. This strategy might have contributed to a lower response rate compared with a previous study in the same health system that also used IVR to contact patients shortly after an office visit. ${ }^{10}$ Cessation resources offered to smokers primarily assist those who are ready to make a quit attempt, yet most smokers are not ready do so at any given time. This is supported by the fact that three-quarters of this study's enrollees planned to quit in the next 30 days. One strategy to improve the overall yield of IVR would be supplement it with other outreach modalities such as text messaging, EHR portals, or email. ${ }^{21}$ Another approach to improve the response to outreach efforts overall would be to adopt a chronic disease model and reach out to a population of smokers to offer treatment on a regular basis. Outreach to smokers regardless of their motivation to quit may be a helpful population health strategy, as smokers without motivation to quit have still been shown to increase quit attempts when provided with treatment such as NRT. ${ }^{22,23}$

This study has several limitations. First, sample sizes were limited by the overall low participation rate, reducing statistical power to detect small differences. Second, the low response rate to follow-up surveys $(54.7 \%)$ depressed cessation rates calculated under the assumption that non-responders are smokers. Third, we did not biochemically validate selfreported nonsmoking, but this is consistent with recommendations for population-based interventions. ${ }^{24}$ Fourth, we did not measure the cost of each strategy, and therefore cannot determine which strategy is most cost-effective. Fifth, this study was

Table 3 Documented Use of Evidence-Based Cessation Treatment in the Two Intervention Groups

\begin{tabular}{|c|c|c|c|c|c|c|}
\hline & \multirow{2}{*}{$\begin{array}{l}\text { Internal care } \\
\text { coordination } \\
\text { (ICC) } \\
n=79\end{array}$} & \multirow{2}{*}{$\begin{array}{l}\begin{array}{l}\text { External } \\
\text { community } \\
\text { referral (ECR) }\end{array} \\
n=78\end{array}$} & \multirow{2}{*}{$\begin{array}{l}\begin{array}{l}\text { Usual } \\
\text { care (UC) }\end{array} \\
n=76\end{array}$} & \multicolumn{3}{|l|}{$P$ value } \\
\hline & & & & ICC vs. UC & ECR vs. UC & ICC vs. ECR \\
\hline $\begin{array}{l}\text { Any evidence-based smoking cessation treat- } \\
\text { ment }\end{array}$ & $60(76.0 \%)$ & $39(50.0 \%)$ & $26(34.2 \%)$ & $<0.001$ & 0.05 & 0.001 \\
\hline $\begin{array}{l}\text { Use of both evidence-based tobacco cessation } \\
\text { counseling and medication }\end{array}$ & $32(40.5 \%)$ & $24(30.8 \%)$ & $4(5.3 \%)$ & $<0.001$ & $<0.001$ & 0.20 \\
\hline Any counseling & $40(50.6 \%)$ & $33(42.3 \%)$ & $7(9.2 \%)$ & $<0.001$ & $<0.001$ & 0.30 \\
\hline In-person counseling at MGH Health Center & $18(22.8 \%)$ & $3(3.8 \%)$ & $7(9.2 \%)$ & 0.02 & 0.20 & $<0.001$ \\
\hline Telephone counseling by Quitline & $12(15.2 \%)$ & $33(41.8 \%)$ & $0(0 \%)$ & $<0.001$ & $<0.001$ & $<0.001$ \\
\hline $\begin{array}{l}\text { Telephone counseling by MGH Tobacco } \\
\text { Care Coordinator }\end{array}$ & $29(36.7 \%)^{*}$ & $-*$ & $-*$ & - & - & - \\
\hline Any medication & $52(65.8 \%)$ & $30(38.5 \%)$ & $23(30.3 \%)$ & $<0.001$ & 0.28 & $<0.001$ \\
\hline Nicotine replacement therapy (any) & $44(55.7 \%)$ & $28(35.9 \%)$ & $18(23.7 \%)$ & $<0.001$ & 0.10 & 0.01 \\
\hline Nicotine patch & $30(30.8 \%)$ & $10(12.8 \%)$ & $15(19.7 \%)$ & 0.01 & 0.24 & $<0.001$ \\
\hline Nicotine lozenge & $12(15.2 \%)$ & $0(0.0 \%)$ & $5(6.6 \%)$ & 0.09 & 0.03 & $<0.001$ \\
\hline Nicotine gum & $10(12.7 \%)$ & $4(5.1 \%)$ & $2(2.6 \%)$ & 0.02 & 0.68 & 0.10 \\
\hline Nicotine inhaler & $11(13.9 \%)$ & $1(1.3 \%)$ & $1(1.3 \%)$ & 0.003 & 1.0 & 0.003 \\
\hline Bupropion & $12(15.2 \%)$ & $4(5.1 \%)$ & $8(10.5 \%)$ & 0.39 & 0.21 & 0.04 \\
\hline Varenicline & $12(15.2 \%)$ & $4(5.1 \%)$ & $4(5.3 \%)$ & 0.04 & 1.0 & 0.04 \\
\hline
\end{tabular}

* Only participants in the internal care coordination group had access to the MGH Tobacco Coordinator 
limited to smokers rather than all tobacco users. While most tobacco users are cigarette smokers, findings may not be generalizable to users of other tobacco products. Sixth, not all state quitlines provide treatment to all tobacco users in the state, and therefore quitline-related findings may not be generalizable to all states. Finally, because provision of smoking cessation medication was derived from EHR and quitline records, we cannot know if participants used the medications.

In summary, a population health strategy of outreach to smokers using automated phone calls was feasible though it had modest reach. Both interventions, linking participants to either health system-based or community resources, outperformed usual care in providing tobacco cessation treatment to smokers who responded to the outreach. Future work should aim to improve population reach of efforts based outside of clinical encounters and test the effect on smoking cessation rates. Such proactive outreach to smokers represents a potentially cost-effective strategy for improving patient uptake of both health system-based and community-based smoking cessation resources, complementing clinicians' office-based care.

Corresponding Author: Sara Kalkhoran, MD, MAS; Division of General Internal Medicine, Department of Medicine Massachusetts General Hospital, Boston, MA, USA (e-mail: skalkhoran@mgh. harvard.edu).

Funding Information This study was financially supported by the Pfizer Independent Grants for Learning and Change (IGLC). IGLC grant recipients were selected independent of Pfizer, by the Smoking Cessation Leadership Center at the University of California, San Francisco. Dr. Kalkhoran is financially supported by the National Heart, Lung, and Blood Institute (NHLBI) of the National Institutes of Health (K23HL136854).

\section{Compliance with Ethical Standards:}

Conflict of Interest: Drs. Rigotti and Kalkhoran receive royalties from UpToDate, Inc. Dr. Rigotti has been an unpaid consultant to Pfizer, Inc. and a paid consultant to Achieve Life Sciences. No other authors have any conflicts of interest to disclose.

Disclaimer: The funders had no role in the design and conduct of the study; collection, management, analysis, and interpretation of the data; preparation, review, or approval of the manuscript; and decision to submit the manuscript for publication.

\section{REFERENCES}

1. US Department of Health and Human Services. The health consequences of smoking - 50 years of progress: a report of the Surgeon General. Atlanta, GA: US Department of Health and Human Services, Centers for Disease Control and Prevention, National Center for Chronic Disease Prevention and Health Promotion, Office on Smoking and Health. $2014 ; 17$.

2. Jamal A, King BA, Neff LJ, Whitmill J, Babb SD, Graffunder CM. Current cigarette smoking among adults - United States, 2005-2015. MMWR Morb Mortal Wkly Rep. 2016;65(44):1205-1211.

3. Fiore MC, Bailey WC, Cohen SJ, et al. Treating tobacco use and dependence: clinical practice guideline. Rockville, MD: US Department of Health and Human Services. 2000;00-0032.
4. Babb S, Malarcher A, Schauer G, Asman K, Jamal A. Quitting Smoking Among Adults - United States, 2000-2015. MMWR Morb Mortal Wkly Rep. 2017;65(52): 1457-1464.

5. Tong EK, Strouse R, Hall J, Kovac M, Schroeder SA. National survey of U.S. health professionals' smoking prevalence, cessation practices, and beliefs. Nicotine Tob Res. 2010;12(7):724-733.

6. Joseph A, Fu S. Proactive outreach strategies to connect smokers with tobacco cessation treatment. JAMA Intern Med. 2015;175(2):226-227.

7. Murray RL, Coleman T, Antoniak M, et al. The effect of proactively identifying smokers and offering smoking cessation support in primary care populations: a cluster-randomized trial. Addiction. 2008;103(6):998-1006; discussion 1007-1008.

8. Fu SS, van Ryn M, Nelson D, et al. Proactive tobacco treatment offering free nicotine replacement therapy and telephone counselling for socioeconomically disadvantaged smokers: a randomised clinical trial. Thorax. 2016;71(5):446-453.

9. Fu SS, van Ryn M, Sherman SE, et al. Proactive tobacco treatment and population-level cessation: a pragmatic randomized clinical trial. JAMA Intern Med. 2014;174(5):671-677.

10. Haas JS, Linder JA, Park ER, et al. Proactive tobacco cessation outreach to smokers of low socioeconomic status: a randomized clinical trial. JAMA Intern Med. 2015;175(2):218-226.

11. Rigotti NA, Bitton A, Kelley JK, Hoeppner BB, Levy DE, Mort E. Offering population-based tobacco treatment in a healthcare setting: a randomized controlled trial. Am J Prev Med. 2011;41(5):498-503.

12. Rogers ES, Fu SS, Krebs $\mathbf{P}$, et al. Proactive tobacco treatment for smokers using Veterans Administration Mental Health Clinics. Am J Prev Med. 2018;54(5):620-629.

13. North American Quitline Consortium. What is a Quitline. http://www. naquitline.org/?page=whatisquitline. Accessed March 5, 2019.

14. McDaniel AM, Benson PL, Roesener GH, Martindale J. An integrated computer-based system to support nicotine dependence treatment in primary care. Nicotine Tob Res. 2005; 7 Suppl 1:S57-66.

15. National Cancer Institute. SmokefreeTXT; https://smokefree.gov/ smokefreetxt. Accessed March 5, 2019.

16. Baker TB, Piper ME, McCarthy DE, et al. Time to first cigarette in the morning as an index of ability to quit smoking: implications for nicotine dependence. Nicotine Tob Res. 2007;9(Suppl 4):S555-570.

17. Sherman SE, Krebs P, York LS, et al. Telephone care co-ordination for tobacco cessation: randomised trials testing proactive versus reactive models. Tob Control. 2018;27(1):78-82.

18. Bodenheimer T, Wagner EH, Grumbach $\mathbf{K}$. Improving primary care for patients with chronic illness. JAMA. 2002;288(14):1775-1779.

19. Bodenheimer $\mathbf{T}$, Wagner $\mathbf{E H}, \mathbf{G r u m b a c h ~} \mathbf{K}$. Improving primary care for patients with chronic illness: the chronic care model, Part 2. JAMA. 2002;288(15):1909-1914.

20. Stellefson M, Dipnarine $\mathbf{K}$, Stopka C. The chronic care model and diabetes management in US primary care settings: a systematic review. Prev Chronic Dis. 2013;10:E26.

21. Zhu SH, Lee $\mathbf{M}$, Zhuang YL, Gamst A, Wolfson T. Interventions to increase smoking cessation at the population level: how much progress has been made in the last two decades? Tob Control. 2012;21(2): 110-118.

22. Carpenter MJ, Hughes JR, Gray KM, Wahlquist AE, Saladin ME, Alberg AJ. Nicotine therapy sampling to induce quit attempts among smokers unmotivated to quit: a randomized clinical trial. Arch Intern Med. 2011;171(21):1901-1907.

23. Jardin BF, Cropsey KL, Wahlquist AE, et al. Evaluating the effect of access to free medication to quit smoking: a clinical trial testing the role of motivation. Nicotine Tob Res. 2014;16(7):992-999.

24. Biochemical verification of tobacco use and cessation. Nicotine Tob Res. 2002;4(2):149-159.

Publisher's Note Springer Nature remains neutral with regard to jurisdictional claims in published maps and institutional affiliations. 